\title{
Neuro QoL Cognitive Function Subdomain
}

National Cancer Institute

\section{Source}

National Cancer Institute. Neuro QoL Cognitive Function Subdomain. NCI Thesaurus.

Code 137842

The collection of Neuro-QoL item scales that assess perceived difficulties in cognitive abilities related to both executive function and general concerns. 